\title{
Cervical collar presence and prandial aspiration among trauma patients
}

\author{
Bhattacharya $\mathrm{B}^{1 *}$, Davis KA ${ }^{1}$, Young $\mathrm{NO}^{2}$ and Leder $\mathrm{SB}^{1}$ \\ ${ }^{1}$ Department of Surgery, Section of Trauma, Surgical Critical Care, and Surgical Emergencies, Yale School of Medicine, New Haven, USA \\ ${ }^{2}$ Department of Surgery, Section of Otolaryngology, Yale School of Medicine,New Haven, USA
}

\begin{abstract}
Background: Prandial pulmonary aspiration is a leading cause of nosocomial infection after traumatic injury.One variable thought to increase aspiration risk is the wearing of a cervical collar. The purposes of this study were to investigate aspiration and aspiration risk prior to initiating oral alimentation among trauma patients requiring a cervical collar.
\end{abstract}

Methods: Study design was a single group $(\mathrm{n}=22)$ referred case series with multiple blinded judges.Inclusion criteria were $\geq 18$ years of age, need for a cervical collar, nil per os status, normal hemodynamics, and completion of either the Yale Swallow Protocol to determine aspiration risk or, if failed, a fiber optic endoscopic evaluation of swallowing (FEES) to determine aspiration status. Testing was done under two conditions:Collar ON followed by Collar OFF.Eating and drinking success were accessed from each patient's electronic medical record one day after testing and again at hospital discharge.Care providers were blinded to the study's purpose.

Results: All 22 (100\%) patients exhibited the same pattern of swallowing success under the collar ON and collar OFF conditions.All were eating and drinking successfully at both one day after testing and at discharge (mean=12.9 days; range=1-73 days).

Conclusions: Wearing a cervical collar was not associated with the incidence of aspiration, aspiration risk, or prandial aspiration among trauma patients. Patient's cognitive status and oral motor functioning are crucial in determining candidacy for safe participation in and ultimate success of swallow screening.

\section{Introduction}

Prandial pulmonary aspiration is a leading cause of nosocomial infection after traumatic injury [1,2] making early identification ofaspiration and aspiration riska high priority before beginning oral alimentation. It has been conjectured that cervical bracing alters certain biomechanical aspects of the oropharyngeal swallow due to unnatural neck hyper extension and immobility [3]. However, the association between cervical bracing and swallowing is based upon limited data [3-6].

The absence of data regarding the association between the need for a cervical collar and the incidence of prandial aspiration among general trauma patients warrants a prospective investigation. The purposes of this study were to investigate aspiration and aspiration risk prior to initiating oral alimentation in acute-care trauma patients requiring a cervical collar. Our study hypothesized that wearing a cervical collar would notbe associatedwithprandial aspiration.

\section{Methods}

\section{Subjects}

This study was approved by the Human Investigation Committee, Yale School of Medicine. In a prospective manner, 22 adult trauma patients were referred to speech-language pathology for swallowing testing by the attending trauma surgeon, physician assistant, or advanced practice nurse practitioner participated (Table 1). Inclusion criteria were $\geq 18$ years of age, need for a cervical collar, nil per os status, normal hemodynamics, stable at time oftesting, appropriate mental status and cognitive function for safe participation evidenced by answering orientation questions and following single-step verbal directions, and completion of either the Yale Swallow Protocol [7] to determine aspiration risk or afiberoptic endoscopic evaluation of swallowing (FEES) [8,9] to determine aspiration status.All patients wore an Aspen (Vista) collar (Aspen Medical Products, Irvine, CA 92618) that controls the head with padded mandibular and occipital supports attached to thoracic plates.

\section{Procedures}

The study design was a single group referred case series with multiple blinded judges. Swallow testing was done under two conditions. First, with the patient wearing the cervical collar $(\mathrm{ON})$ and then, after appropriate physical exam and clearance of the patient's cervical spine, swallow testing was performed a second time with the cervical collar removed (OFF). Eating and drinking success was accessed from each patient's electronic medical record one day after testing and again at hospital discharge. Care providers were blinded to the study's purpose.

\section{Yale swallow protocol}

The Yale Swallow Protocol [7] is a valid and reliable swallow screen

Correspondence to: Bishwajit Bhattacharya, Department of Surgery, Section of Trauma, Surgical Critical Care and Surgical Emergencies, Yale University School of Medicine, USA, E-mail: Bishwajit.bhattacharya@yale.edu

Key words: deglutition, deglutition disorders, dysphagia, aspiration risk, trauma

Received: October 22, 2016; Accepted: November 08, 2016; Published: November 12, 2016 
to determine aspiration risk and make diet recommendations for acute care ICU patients[10].It is composed of a brief cognitive evaluation [11], oral mechanism examination [12], and a 3-ounce water swallow challenge [13]. Passing allows for an oral diet to begin, usually regular consistency if dentition is adequate and puree if edentulous, while failing results in referral for instrumental testing to determine aspiration status.

\section{Fiber optic endoscopic evaluation of swallowing (FEES)}

The standard FEES protocol was followed with slight modifications $[8,9]$. Briefly, each naris was examined visually and the scope passed through the most patent naris without administration of a topical anesthetic or vasoconstrictor to the nasal mucosa, thereby eliminating any potential adverse anesthetic reaction and assuring the endoscopist of a safe physiologic examination [14]. The base of tongue, pharynx, and larynx were viewed and swallowing was evaluated directly with six food boluses of approximately 5-10 cc volume each. Patients were encouraged to feed themselves, with assistance as needed, i.e., liquid with a straw or cup and puree with a spoon. All patients were allowed to swallow spontaneously, i.e., without a verbal command to swallow [15].FEES equipment consisted of a flexible fiberopticrhinolaryngosope (KayPentax, Lincoln Park, NJ 07035, model VNL-117OK), light source (KayPentax, model EPK-1000), and a digital swallow workstation (KayPentax, model 7200).

The first food challenge consisted of three boluses of puree consistency (yellow pudding) followed by three thin liquid boluses (white, fat free, skim milk), as these colors have excellent contrast with pharyngeal and laryngeal mucosa [16]. A solid food challenge, i.e., graham cracker, was given only if the patient was dentate Aspiration was defined as entry of material into the airway below the level of the true vocal folds [17]. A functional swallow was without aspiration and allowed for resumption of oral alimentation.

A pre-existing protocol directed nursing to discontinue the patient's oral diet and re-consult speech-language pathology if overt signs of aspiration, e.g., increased cough, increased temperature, or signs/symptoms of an upper respiratory infection, were noted during or after meal-timeseither within 24 hours or at any subsequent time before hospital discharge.Thin liquids and puree consistency foods were recommended for edentulous patients and thin liquids, puree, and solid food consistencies for dentate patients.

\section{Reliability testing}

Confirmatory intra-rater and inter-rater agreements of FEES findings were performed on $20 \%$ of the sample, i.e., binary decisions of presence or absence of aspiration on each swallow for each participant. One speech-language pathologist and one otolaryngologist, with $>10$ years of experience in interpreting FEES results, independently and blindly reviewed the individual swallows on a digital swallowing workstation.Using real-time analysis with repeat viewing as needed, both intra-rater and inter-rater agreement ratings were $100 \%$ for identification of presence or absence of tracheal aspiration during FEES.

\section{Results}

A referred sampleof 22 adult trauma patients from a large, urban, tertiary care, teaching hospital participated. Table 1 shows

Table 1. Participant characteristics.

\begin{tabular}{|c|c|c|c|}
\hline \multirow{2}{*}{$\begin{array}{c}\text { Participant No./Gender/Age } \\
\text { (years) }\end{array}$} & \multirow[t]{2}{*}{ Etiology/Diagnosis } & \multicolumn{2}{|c|}{ Testing with Cervical Collar First ON and Second OFF } \\
\hline & & $\begin{array}{l}\text { Yale swallow protocol diet } \\
\text { recommendations }\end{array}$ & FEES ${ }^{\mathrm{a}}$ results and diet recommendations \\
\hline $1 / \mathrm{M} / 90$ & Fall/Rib fractures & Failed & Modified diet nectar-like thick liquids/puree \\
\hline $2 / \mathrm{F} / 83$ & Fall/Left parieto- temporal SDH ${ }^{\mathrm{b}}$ & Failed & Nil per os with $\mathrm{NJ}$ tube feedings \\
\hline $3 / \mathrm{F} / 93$ & Fall/Frontoparietal hemorrhage & Failed & $\begin{array}{l}\text { Weak/delayed oropharyngeal swallow/Thin liquid and } \\
\text { puree diet }\end{array}$ \\
\hline $4 / \mathrm{M} / 64$ & $\mathrm{C}_{4-5} \mathrm{ACDF}^{\mathrm{c}} /$ Spinal cord compression & Failed & Modified diet nectar-like thick liquids/puree \\
\hline $5 / \mathrm{F} / 48$ & Suicide attempt/Hanging & Passed; Regular diet & - \\
\hline $6 / \mathrm{M} / 59$ & Assault/Stabbing & Passed; Regular diet & - \\
\hline 7/M/19 & Trauma/MVC & Passed; Regular diet & - \\
\hline $8 / \mathrm{M} / 34$ & Suicide attempt/Jumped out of window & Passed; Regular diet & - \\
\hline 9/M/74 & MVC & Passed; Puree diet & - \\
\hline $10 / \mathrm{M} / 61$ & Found down & Passed; Puree diet & - \\
\hline $11 / \mathrm{F} / 82$ & MVC & Passed; Regular diet & - \\
\hline $12 / \mathrm{M} / 28$ & MVC & Passed; Regular diet & - \\
\hline $13 / \mathrm{F} / 51$ & MVC & Passed; Regular diet & - \\
\hline $14 / \mathrm{F} / 72$ & Fall at home & Passed; Regular diet & - \\
\hline $15 / \mathrm{F} / 52$ & MVC & Passed; Regular diet & - \\
\hline $16 / \mathrm{F} / 22$ & MVC & Passed; Regular diet & - \\
\hline $17 / \mathrm{M} / 48$ & Bicycle crash & Passed; Regular diet & - \\
\hline $18 / \mathrm{M} / 52$ & Altercation & Passed; Regular diet & - \\
\hline $19 / \mathrm{M} / 29$ & Fall/Balcony & Passed; Regular diet & - \\
\hline $20 / \mathrm{F} / 92$ & Fall at home & Passed; Regular diet & - \\
\hline $21 / \mathrm{M} / 57$ & $\begin{array}{l}\text { Fall/Right } \quad \text { temporal subarachnoid } \\
\text { hemorrhage }\end{array}$ & Passed; Regular diet & - \\
\hline $22 / \mathrm{F} / 60$ & Fall/Seizures & Passed; Puree diet & - \\
\hline
\end{tabular}

Totals: 12M (Mean age: 51.3; Range: 19-90 y); 10F (Mean age: 65.5; Range: 22-93 y)

${ }^{a} \mathrm{FEES}=$ Fiberoptic endoscopic evaluation of swallowing

${ }^{\text {bSDH}}=$ Subdural hematoma

${ }^{\mathrm{C}} \mathrm{ACDF}=$ Anterior cervical discectomy and fusion

${ }^{\mathrm{d}} \mathrm{MVC}=$ Motor vehicle crash 
participantdemographics, diagnoses, swallow testing results, and diet recommendations from the Yale Swallow Protocol and FEES.

All $22(100 \%)$ patients exhibited the same pattern of swallowing success under the collar ON and collar OFF conditions. Eighteen patients passed the Yale Swallow Protocol and were successful with either a regular $(n=16)$ or puree $(n=2)$ consistency diet. Three patients failed the Yale Swallow Protocol and required FEES to determine safe oral diet recommendations, e.g., modified diet of nectar-like thickened liquids and puree foods.One patient failed the Yale Swallow Protocol and exhibited aspiration on FEES and, therefore, remained nil per os with enteral tube feedings.

All patients recommended for oral alimentation were eating and drinking successfully at both one day after testing and at discharge (mean=12.9 days; range=1-73 days), i.e., neither the speech-language pathology nor nursing services identified any patient exhibiting overt signs of aspiration.

\section{Discussion}

The presence of a cervical collardid not increase the incidence of aspiration risk orprandial aspiration in acute-care trauma patients meeting the study's inclusion criteria.This agrees with previous findings that aspiration did not occur with cervical bracing in young, normal, healthy volunteers[5].

The present studysupports the findings of successful oral alimentation at one day follow-up in intensive care unit [10] patients andfive-day follow-up in general hospital [18] patients (who did not require a cervical collar). Specifically, when the patient remained medically and neurologically stablesuccessful swallowing was maintained whenthe Yale Swallow Protocol was passed or there was a functional swallow based on FEES.

It is not uncommon for trauma patients who require a cervical collar to exhibit some degree of altered mental status.Knowledge of the patient's cognitive status and oral motor functioning, both provided by the Yale Swallow Protocol [7], are crucial in determining candidacy for safe participation in and ultimate success of swallow screening. The ability to answerorientation questions and follow single-step verbal commands correctly [11] as well as demonstrate functional lingual range of motion [12] provides information on odds of potential prandial aspirationriskprior to swallow testing. The clinician should use this information in the selection of candidates deemed appropriate for swallow screening to optimize swallowing success.

If a reliable and validated swallow screen [7] is failed, objective instrumental endoscopic or video fluoroscopic testing is recommended for acute-care trauma patients requiring a cervical collar. FEES is the test of choice as it is patient friendly, repeatable, performed at bedside, does not require transport to a radiology suite, and uses regular food [9]. A video fluoroscopic swallow study is recommended only if transnasal endoscopy is contra-indicated, e.g., nasal or facial trauma, or impossible to perform, e.g., extreme agitation or combativeness.

\section{Study strengths and limitations}

Strengths of this study include a heterogeneous referred sample for swallowing testing, blinded data acquisition, and 100\% interrater reliability for the outcome variable of aspiration.Limitations are use of one type of cervical collar, i.e., Aspen (Vista). Future research investigating other cervical bracing devices, e.g., sternal occipital mandibular immobilizer, halo-ring, Philadelphia collar, and Minerva, would be of interest.

\section{Conclusions}

Presence of a cervical collardoes not increase incidence of aspiration risk orprandial aspirationin the acute-care trauma patient. Knowledge of the patient's cognitive status and oral motor functioning, both provided by the Yale Swallow Protocol [7], are crucial in determining candidacy for safe participation in and ultimate success of swallow screening. Patients who pass swallow screening or dysphagia testing and remain medically and neurologically stable continue to eat and drink successfully throughout their hospital course until discharge. If a reliable and validated swallow screen for aspiration risk is failed, instrumental endoscopic or video fluoroscopic swallow testingis necessary for acute-care trauma patients who require a cervical collarto determine aspiration status and when to make appropriate, safe, and timely diet recommendations prior to beginning oral alimentation.

\section{Conflict of interest}

None of the authors has any conflict of interest to declare.

\section{References}

1. Yeung L, Miraflor E, Strumwasser A, Sadeghi P, Victorino GP (2012) Does gastric volume in trauma patients identify a population at risk for developing pneumonia and poor outcomes? J Surg Res 178: 874-878. [Crossref]

2. Hyllienmark P, Brattström O, Larsson E, Martling CR, Petersson J, et al. (2013) High incidence of post-injury pneumonia in intensive care-treated trauma patients. Acto Anaesthesiol Scand 57: 848-854

3. Morishima N, Ohota K, Miura Y (2005) The influences of Halo-vest fixation and cervical hyperextension on swallowing in healthy volunteers. Spine (Phila Pa 1976) 30: E179-E182. [Crossref]

4. Houghton DJ, Curley JW (1996) Dysphagia caused by a hard-cervical collar. $\mathrm{Br} J$ Neurosurg 10: 501-502. [Crossref]

5. Stambolis V, Brady S, Klos D, Wesling M, Fatianov T, et al. (2003) The effects of cervical bracing upon swallowing in young, normal, healthy volunteers. Dysphagia 18: 39-45. [Crossref]

6. Shem K, Castillo K, Wong S, Chang J (2011) Dysphagia in individuals with tetraplegia: incidence and risk factors. J Spinal Cord Med 34: 85-92. [Crossref]

7. Leder SB, Suiter DM (2014) The Yale Swallow Protocol: An Evidenced-Based Approach to Decision Making. Springer Science+Business Media, NY.

8. Langmore SE, Schatz K, Olson N (1991) Endoscopic and videofluoroscopic evaluation of swallowing and aspiration. Ann Otol Rhinol Laryngol 100: 678-681. [Crossref]

9. Leder SB, Murray JT (2008) Fiberoptic endoscopic evaluation of swallowing. Phys Med Rehabil Clin N Am 19: 787-801. [Crossref]

10. Leder SB, Suiter DM, Warner HL, Kaplan LJ (2011) Initiating safe oral feeding in critically ill intensive care and step-down unit patients based on passing a 3-ounce (90 milliliters) water swallow challenge. $J$ Trauma 70: 1203-1207.

11. Leder SB, Suiter DM, Warner HL (2009) Answering orientation questions and following single-step verbal commands: Effect on aspiration status. Dysphagia 24: 290-295.

12. Leder SB, Suiter DM, Murray J, Rademaker AW (2013) Can an oral mechanism examination contribute to the assessment of odds of aspiration? Dysphagia 28: 370374

13. Suiter DM, Leder SB (2008) Clinical utility of the 3-ounce water swallow test. Dysphagia 23: 244-250. [Crossref]

14. Leder SB, Ross DA, Briskin KB, Sasaki CT (1997) A prospective, double-blind, randomized study on the use of a topical anesthetic, vasoconstrictor, and placebo during transnasal flexible fiberoptic endoscopy. J Speech Lang Hear Res 40: 1352 1357. [Crossref]

15. Daniels SK, Schroeder MF, DeGeorge PC, Corey DM, Rosenbek JC (2007) Effects of verbal cue on bolus flow during swallowing. Am J Speech Lang Pathol 16: 140-147. [Crossref]

16. Leder, Acton LM, Lisitano HL, Murray JT (2005) Fiberoptic endoscopic evaluation 
Bhattacharya B (2016) Cervical collar presence and prandial aspiration among trauma patients

of swallowing (FEES) with and without blue-dyed food. Dysphagia 20: 157-162. [Crossref]

17. Logemann, JA. Evaluation and Treatment of Swallowing Disorders. 2nd ed. Austin, TX: Pro-Ed; 1998
18. Leder SB, Suiter DM (2014) Five days of successful oral alimentation for hospitalized patients based upon passing the Yale Swallow Protocol. Ann Otol Rhinol Laryngol 123 609-613. [Crossref]

Copyright: (C2016 Bhattacharya B. This is an open-access article distributed under the terms of the Creative Commons Attribution License, which permits unrestricted use, distribution, and reproduction in any medium, provided the original author and source are credited. 